Artigo Original

\title{
Avaliação dos níveis de monóxido de carbono em funcionários de um hospital geral
}

Evaluation of the carbon monoxide levels in staff of a general hospital

Tânia Cristina Malezan Fleig ${ }^{1,}$ André Luiz Kunzler ${ }^{1}$ Christian Portz ${ }^{1}$ Guilherme Dionir Back ${ }^{1}$ Paloma de Borba Schneiders ${ }^{1}$ Elisabete Antunes San Martin ${ }^{1}$ Andréa Lúcia Gonçalves da Silva ${ }^{1}$

${ }^{1}$ Universidade de Santa Cruz do Sul- UNISC, Santa Cruz do Sul, RS.

Submissão: 11/08/2016

Aceite: 06/09/2016

andreag@unisc.br

\section{RESUMO}

Justificativa e Objetivos: A exposição ao monóxido de carbono (CO) pode ser prejudicial para a saúde humana e os primeiros efeitos da intoxicação podem passar despercebidos. Nosso objetivo foi avaliar acapacidade funcional e força muscular respiratória de funcionários de um hospital geral frente à inalação de monóxido de carbono. Métodos: estudo de delineamento transversal, com amostragem de conveniência. Utilizou-se como instrumentos de avaliação: roteiro de investigação clínica; MicroCO para análise da concentração $\mathrm{CO}$, com ponto de corte de $\mathrm{CO}$ para não fumantes entre 1-6ppm, fumantes leves7-10ppm, fumantes moderados 11-20ppm e fumantes pesados valores $>20 \mathrm{ppm}$; Manovacuometria para determinar a força muscular respiratória ( $\mathrm{PI}_{\text {máx }}$ e $\mathrm{PE}_{\text {máx }}$ ); Teste de Caminhada de Seis minutos (TC6m) para avaliar a capacidade funcional. A análise estatística foi realizada pelo teste de correlação de Pearson para verificar a associação das variáveis. Resultados: Participaram do estudo 14 sujeitos voluntários ( 8 homens), idade média de $35,14 \pm 8,76$ anos, atuantes na manutenção $(n=5)$; lavanderia $(n=6)$; cozinha $(n=1)$; engenharia $(n=2)$ do hospital. Para tabagismo ativo apenas 3 sujeitos ficaram acima do ponto de corte $(\mathrm{CO}>6 \mathrm{ppm})$, sendo considerados fumantes ativos. A PI máx $\left(99,50 \pm 27,37 \%\right.$ predito) e $\mathrm{PE}_{\text {máx }}(105,64 \pm 21,39 \%$ predito) encontram-se dentro dos parâmetros de normalidade. A capacidade funcional dos indivíduos fumantes (TC6m=71\%predito), com alto nível CO $(17 \mathrm{ppm})$, ficou aquém do valor basal quando comparados com os indivíduos não fumantes $(\mathrm{CO}=0,9 \mathrm{ppm}$; TC6m=92,6\% predito). Correlação moderada e inversa foi observada entre TC6m e CO $(\mathrm{r}=-0,577, \mathrm{p}=0,031)$. Conclusão: Funcionários adultos de um hospital geral,com força muscular respiratória preservada e maiores níveis de $\mathrm{CO}$ exalados apresentam pior desempenho funcional no TC6m. 
DESCRITORES: Força muscular. Monóxido de Carbono. Saúde do Trabalhador. Saúde Pública.

\begin{abstract}
Backgrounds and Objective: Exposure to carbon monoxide (CO) can be harmful to human health and the first effects of intoxication may go unnoticed. Our objective was to evaluate the functional capacity and respiratory muscle strength of staff of a general hospital opposite the inhalation of carbon monoxide. Methods: cross-sectional study with a convenience sample. It was used as evaluation tools: clinical research roadmap; the MicroCO for analysis of CO concentration, CO cutoff for non-smokers among 1$6 \mathrm{ppm}$, light smokers 7-10ppm, moderate smokers 11-20ppm and heavy smokers >20ppm; the manometer to determine the respiratory muscle strength (MIP and MEP); Six minute walk test (6MWT) to evaluate functional capacity. Statistical analysis was performed hair Pearson correlation test to verify the association of variables. Results: The study included 14 volunteer subjects (08 men), mean age 35.14 $\pm 8,76$ years, working in maintenance $(n=5)$; laundry $(n=6)$; kitchen $(n=1)$; engineering $(n=2)$ of the hospital. For active smokers only 03 subjects were above the cutoff $(\mathrm{CO}>6 \mathrm{ppm})$ then being considered active smokers. The MIP (99.50 $\pm 27.37 \%$ predicted) and MEP (105.64 $\pm 21,39 \%$ predicted) are within normal parameters. The functional capacity of smokers ( $6 \mathrm{MWT}=71 \%$ of predicted), with high CO (17ppm), underperformed the baseline when compared with nonsmokers $(\mathrm{CO}=0,9 \mathrm{ppm} ; 6 \mathrm{MWT}=92,6 \%$ predicted $)$. Moderate and negative correlation was observed between 6MWT and CO $(\mathrm{r}=-0.577, \mathrm{p}=0.031)$. Conclusion: adult staff of a general hospital with preserved respiratory muscle strength and higher levels of CO exhaled have worse functional performance in the 6MWT.
\end{abstract}

KEYWORDS:Muscle Strength. Carbon Monoxide.Occupational Health.Public Health.

\title{
INTRODUÇÃO
}

A saúde do trabalhador constitui uma área da Saúde Pública que busca a intervenção nas relações entre trabalho e saúde e possui como objetivos a promoção e a proteção da saúde do trabalhador, por meio do desenvolvimento de ações de vigilância quanto aos riscos presentes nos ambientes e condições de trabalho, aos agravos à saúde do trabalhador e a organização e prestação da assistência aos trabalhadores, compreendendo procedimentos de diagnóstico e reabilitação de forma integrada. ${ }^{1,2}$

Os elevados níveis de gases, poeiras e aerossóis presentes no ambiente de trabalho estão associados a uma extensa gama de patologias que acometem o sistema respiratório. A exposição ao monóxido de carbono (CO) pode ser extremamente prejudicial para a saúde humana e os primeiros efeitos da intoxicação podem passar despercebidos e começam a causar hipoxia tecidual resultando em uma variedade de sintomas agudos incluindo dor de cabeça, tonturas, fraqueza, náusea, confusão, desorientação e distúrbios visuais. ${ }^{1,3-6}$ 
O sistema respiratório mantém um importante vínculo entre o organismo humano e o meio ambiente, em particular com o ar e sua composição. Desta forma, os efeitos patológicos causados pela exposição aos poluentes do ar no ambiente ocupacional são influenciados por fatores como: as propriedades químicas dos gases e aerossóis, as características de cada indivíduo, bem como sua herança genética preexistentes e os hábitos de vida como, por exemplo, o tabagismo. ${ }^{1,3}$

O tabagismo é um grave problema de saúde públicasendoconsiderado a principal causa de morte evitável em todo o mundo. No Brasil, 200 mil mortes anuais são causadas pelo tabagismo. No contexto da saúde ocupacional, o tratamento do tabagismo consiste na prevenção do seu início, através de recomendações específicas, numa rotina diária de atenção à saúde. ${ }^{7,8}$ Além disso, o CO presente nos componentes do cigarro pode reduzir os níveis de oxigênio na cóclea e resultar em vasoconstrição, aumento do fluxo sanguíneo, redução do transporte de oxigênio e dificuldade na dissociação da oxihemoglobina. ${ }^{9}$

O medidor de Monóxido de Carbono (MicroCO) é utilizado para medir a concentração de $\mathrm{CO}$ na respiração e calcular o percentual de carboxihemoglobina no sangue, frequentemente utilizado em tratamentos de interrupção do tabagismo, cirurgias de grande porte. Departamentos de emergências, bem como pelos serviços contraincêndio, também tem lançado mão deste instrumento para avaliar o grau de intoxicação. Nos tratamentos de interrupção ao tabagismo, o MicroCO é utilizado pelas finalidades de instrução e para verificação do progresso e conformidade.

Partindo do exposto, nós hipotetizamos que os funcionários de diferentes setores de um Hospital Geral apresentam redução da capacidade funcional em associação com os maiores níveis de CO inalado. Sendo assim, o nosso objetivo foi avaliar capacidade funcional e a força muscular respiratória de funcionários de um Hospital Geral frente à inalaçãode monóxido de carbono.

\section{MÉTODOS}

Estudo de delineamento transversal e caráter quantitativo, composto por amostragem de conveniência. O projeto de pesquisa foi apreciado e aprovado pelo Comitê de Ética em Pesquisa (CEP-UNISC) sob protocolo de $n^{\circ} 2488 / 09$, sendo que a pesquisa foi realizada no Hospital Santa Cruz, de Santa Cruz do Sul - RS. Foram incluídos no estudo funcionários do hospital, de ambos os sexos, maiores de 18 anos, que estavam ativos em atividades laborais, nos setores apontados pelo Serviço Especializado em Engenharia de Segurança e em Medicina do Trabalho (SESMT) do hospital. Estes 
indivíduos concordaram em participar do estudo através do Termo de Consentimento Livre e Esclarecido (TCLE) e responderam ao roteiro de investigação de forma válida, propondo-se a participar das avaliações.Foram excluídos os funcionários que possuíam doenças crônicas (problemas cardiovasculares, cardiorrespiratórios ou câncer)e que não completaram o roteiro de avaliação.

Os participantes foram convidados a responder um roteiro de investigação composto por questões objetivas, pertinentes a hábitos de vida, meio de locomoção e ocupação no local de trabalho. As variáveis analisadasneste estudo foramconcentração de $\mathrm{CO}$, a força muscular respiratória $\left(\mathrm{PI}_{\text {máx }}\right.$ e $\left.\mathrm{PE}_{\text {máx }}\right)$ e a capacidade funcional pelo teste de caminhada de seis minutos (TC6m).

\section{Concentração de CO}

O medidor de Monóxido de Carbono Expirado MicroCo (marca CardinalHealth) baseia-se em uma célula sensora de combustível eletroquímico, a qual trabalha através da reação de $\mathrm{CO}$ em um eletrodo e oxigênio (do ar ambiente) no outro eletrodogerando uma corrente elétrica proporcional à concentração de CO. A saída do sensor é monitorada por um microprocessador, o qual detecta picos de concentração de gás alveolar expirado. Em seguida, o mesmo é convertido em \% de carboxi-hemoglobina (\% $\mathrm{COHb})$, onde níveis elevados de carboxi-hemoglobina são comumente causados por inalação acidental de fumaça/CO ou tabagismo. O MicroCo inclui um temporizador de contagem regressiva como auxílio para medir o tempo no qual realiza-se uma pausa inspiratória antes da exalação. Os resultados são exibidos em um visor claro de LCD em que luzes de aviso são providas para mostrar indicação instantânea sobre o nível de fumaça. ${ }^{10,11}$

A medida do $\mathrm{CO}$ foi feita com o indivíduo em sedestação, com o dorso no espaldar da cadeira, quadril e joelhosem ângulo de $90^{\circ}$ apoiados no solo, realizando uma pausa inspiratória por 20 segundos, enquanto que o pesquisador iniciava a contagem regressiva de 10 segundos. A seguir, com os lábios no bocal descartável do equipamento, o indivíduofoi orientado a realizar expiração lenta e completa. Feito isto, a medida de CO foi registrada no visor do analisador, sendo expressa em ppm, após o término da manobra. Desta forma obteve-se o dado da concentração de monóxido de carbono, seguindo o previsto de caracterização na tabela CO MeasurementTable, sendo consideradas 1-6ppm (Non-Smoker); 7-10 (Light Smoker); 11-20 (Smoker); 20+ (Heavy Smoker). A medida ppm indica a concentração de partículas por milhão de monóxido de carbono. ${ }^{10,11}$ 


\section{Força Muscular Respiratória (FMR)}

A FMR foi avaliada através da manovacuometria digital (MDI ${ }^{\circledR}$, modelo MVD300, Porto Alegre, Brasil), que identifica valores de Pressão Inspiratória máxima ( $\left.\mathrm{PI}_{\text {máx }}\right)$ e Pressão Expiratória máxima ( $\left.\mathrm{PE}_{\text {máx }}\right)$. A PI máx foi obtida após o indivíduo expirar até o volume residual (VR), tendo o examinador conectado o clipe nasal e solicitado a

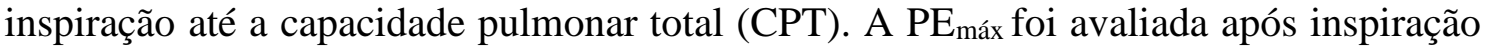
da CPT até o VR contra válvula ocluída. ${ }^{12}$ Para a análise dos dados, foi considerado o maior valor obtido dentre as cinco manobras realizadas, que não diferisse mais que $10 \%$ do segundo maior valor em ordem decrescente. Tais valores foram posteriormente comparados com os descritos na literatura e expressos em percentual do predito. ${ }^{13}$ Ressalta-se que para tais medidas, o paciente permaneceu em sedestação na cadeira ou poltrona.

\section{Teste de Caminhada de Seis Minutos - TC6m}

O Teste de Caminhada de Seis Minutos (TC6m) tem sido utilizado nas avaliações periódicas da capacidade de exercício submáxima em sujeitos que não toleram os testes máximos. Tal teste é de baixo custo, de fácil execução e de boa reprodutibilidade, funcionando como preditor de morbidade e mortalidade. A habilidade de caminhar por uma distância reflete a qualidade de vida e a capacidade de desenvolver as atividades de vida diária. ${ }^{14}$ Visto isto, para avaliar a capacidade funcional dos funcionários foi aplicado o TC6m, onde foi solicitado que percorresse a maior distância em 6 minutos. O teste foi realizado em um corredor plano, sem tráfego, de 30 metros nas dependências do HSC, seguindo o protocolo preconizado pela ATS (2002). ${ }^{12}$ Os resultados de valores preditos foram obtidos através da equação disposta por Enright e Sherril (1998) para homens e mulheres.A Escala Borg foi aplicada a cada minuto. ${ }^{15}$

\section{Análise estatística}

A análise foi realizada através do programa SPSS (Statistical packge for social sciense, versão 20.0). Os dados foram expressos em média e desvio padrão, sendo utilizado teste de correlação de Pearson para verificar a associação das variáveis da Força Muscular Respiratória; Teste de Caminhada de Seis Minutos; Concentração de CO, com nível de significância utilizado de $\mathrm{p} \leq 0,05$.

\section{RESULTADOS}


As características clínicas e sociodemográficas dos sujeitos estudados estão descritas na Tabela 1.

Tabela 1 - Características Clínicas e Sociodemográficas dos Funcionários.

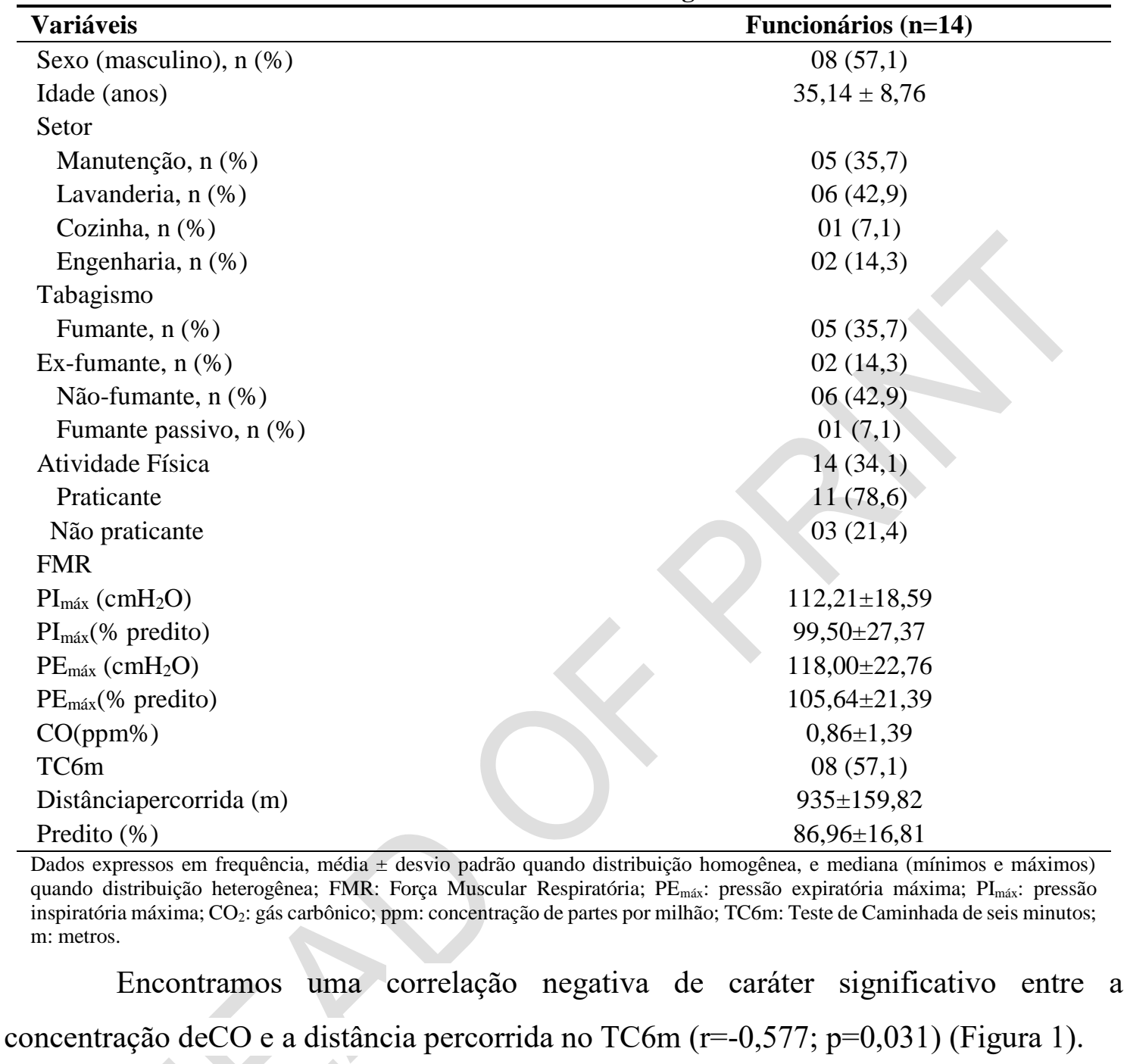




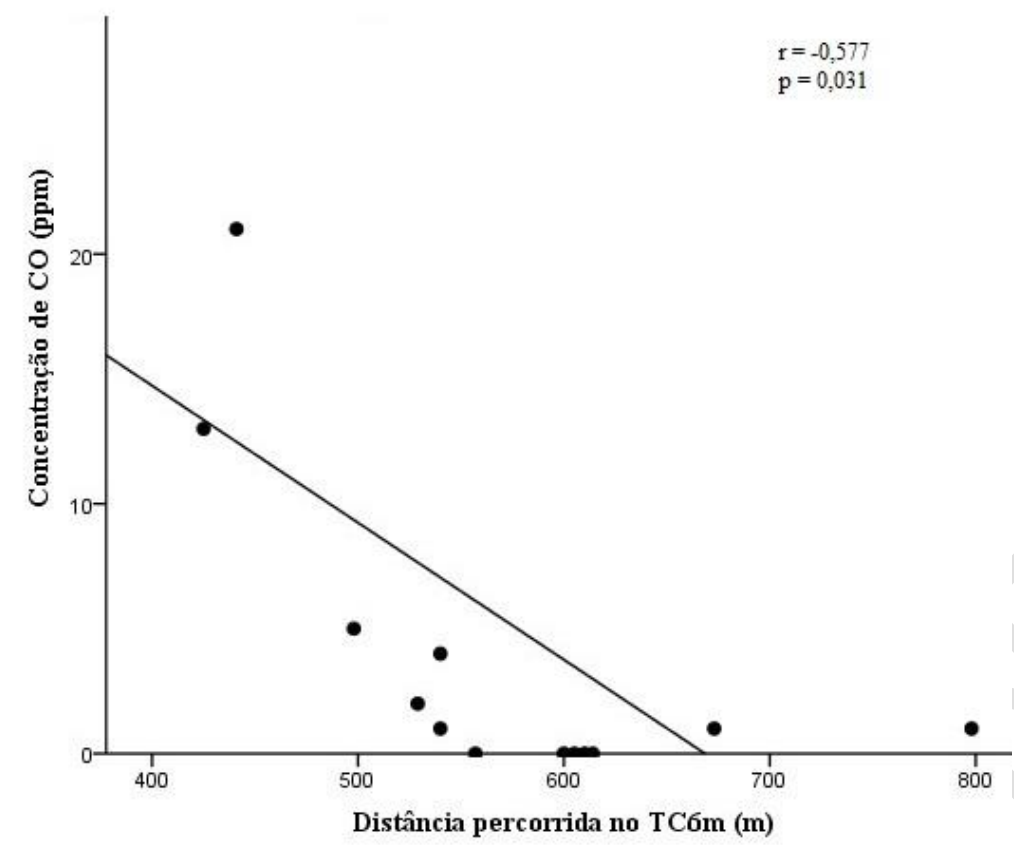

Figura 1 - Relação entre Concentração de CO e capacidade funcional dos funcionários. Correlação entre aConcentração de monóxido de carbono (CO)e distância percorrida no Teste de Caminhada de Seis Minutos (TC6m). Teste de Correlação de Pearson $(\mathbf{p}<0,05)$; ppm: concentração de partes por milhão; m: metros.

Observou-se uma interação importante entre a quantidade de $\mathrm{CO}$ exalado e desempenho na capacidade funcional no TC6m. Os resultados obtidos no desempenho da capacidade funcional dos indivíduos fumantes (TC6m $=71,25 \%$ do predito), com alto nível de $\mathrm{CO}$ exalado (média $\mathrm{CO}=17,25 \mathrm{ppm}$ ), ficaram aquém do valor basal disposto na equação descrita por Moreira, Moraes e Tannus (2001) e Enright e Sherril (1998), quando comparados com os indivíduos não fumantes (média $\mathrm{CO}=0,9 \mathrm{ppm}$; TC6m = 92,6\% do predito). ${ }^{15,16}$

\section{DISCUSSÃO}

Tendo em vista os danos à saúde, que a dependência nicotínica causa, é crescente a demanda por métodos que permitam avaliar com segurança a exposição ao tabaco, principalmente em pacientes em tratamento para cessar o ato de fumar. A medida do monóxido de carbono (CO) tem sido a mais utilizada na prática clínica e em pesquisas para cessação do tabagismo por ser um método não invasivo, de baixo custo e com resultado imediato. ${ }^{17,18}$

Em nosso estudo, identificamos que dentre os funcionários de um hospital geral, apenas um reduzido número $(\mathrm{n}=03)$ apresentou altos níveis de $\mathrm{CO}$ exalado (média $\mathrm{CO}=17 \mathrm{ppm})$. Após ser inalado, o $\mathrm{CO}$ concorre com o oxigênio $\left(\mathrm{O}_{2}\right)$ na corrente sanguínea resultando em uma menor oxigenação de células e tecidos. Com o tempo, isso acarreta o 
envelhecimento precoce do endotélio, da camada de células que formam a parede de vasos e das artérias do corpo humano. Portanto, o CO contém um grande potencial para alterar o transporte de $\mathrm{O}_{2}$ no sangue, já que o $\mathrm{CO}$ compete em vantagem com o $\mathrm{O}_{2}$ pelo local de ligação na molécula de hemoglobina, bem como reduz a liberação de $\mathrm{O}_{2}$ da hemoglobina para os tecidos. ${ }^{19}$ Destaca-se ainda, que a afinidade da hemoglobina ao CO é 37 vezes superior que a do $\mathrm{O}_{2} \cdot{ }^{20}$ Os efeitos do $\mathrm{CO}$ se manifestam mais intensamente nos órgãos e tecidos em que há maior consumo de oxigênio como o coração, o cérebro e o músculo esquelético em atividade. ${ }^{19}$ Desta forma pode-sejustificar o nosso achado no que tange à associação entre os altos níveis de $\mathrm{CO}$ e o pior desempenho dos sujeitos no TC6m, e estes estão de acordo com os achados de Matielo e Gonçalves (1997). ${ }^{20}$

Os níveis de $\mathrm{CO}$ exalado tem relação direta com a capacidade física em consequência um consumo máximo de oxigênio $\left(\mathrm{VO} 2_{\text {máx }}\right)$ menor, ou seja, uma menor capacidade de aproveitamento do oxigênio como energia, com reflexo no menor desempenho da atividade física aeróbica, maior fadiga e falta de fôlego proporcionalmente ao número de cigarros fumados.

Importante ressaltar que o COapresentauma meiavida de aproximadamente seis horas, podendo permanecer no sangue por mais tempo se a fonte produtora do gás se mantiver presente, como poluição ambiental ou exposição ocupacional. No entanto, a causa mais frequente de elevação no $\mathrm{CO}$ decorre do tabagismo ativo ${ }^{21}$.Nos estudos de prevalência de fumantes em determinadas populações, o ponto de corte escolhido está em torno de 5 ou $6 \mathrm{ppm}$. Por outro lado, quando os estudos visam acompanhar fumantes em processo de cessação do tabagismo, necessitando de maior especificidade, utilizam-se valores mais altos, entre 8 e $10 \mathrm{ppm}^{22}$

Mesmo nas diretrizes mais recentes sobre tratamento da adição e do acompanhamento do status tabágico, há discrepância na eleição dos pontos de corte dos vários marcadores bioquímicos. ${ }^{23-25}$ Isto pode ser ressaltado como uma possível limitação do nosso estudo, e também a justificativa para um número limitado de sujeitos com valores elevados de CO exalado. No entanto, nós nos baseamos em outro estudo com amostra brasileira que avaliou a frequência de tabagismo em um hospital geral da cidade de São Paulo, utilizando o CO como marcador, os autores consideraram 6ppm como ponto de corte entre fumantes e não-fumantes. ${ }^{26}$ Santos et al. (2001), também usando valor de corte de CO igual a $6 \mathrm{ppm}$, encontraram sensibilidade de $77 \%$ e especificidade de $96 \%$ em uma população de voluntários (funcionários, usuários e visitantes) do Instituto do Coração em São Paulo. ${ }^{18}$ 
No estudo apresentado pode-se traçar o perfil dos funcionários de um Hospital Geral, dentre as características individuais e a ocupação de trabalho, estabelecendo-se nas variáveis de concentração de $\mathrm{CO}$ o principal elemento de discussão. Conclui-se que os sujeitos que apresentam maiores níveis de $\mathrm{CO}$ exalados apresentam pior desempenho funcional no TC6m quando comparado aos sujeitos com valores de CO exalado abaixo de 6ppm.

AGRADECIMENTOS:SESMT do Hospital Santa Cruz - Santa Cruz do Sul, RS, Brasil.

\section{REFERÊNCIAS}

1. Ministério da Saúde (BR). Portaria n ${ }^{\circ} .95$, de 26 de janeiro de 2001. Norma Operacional da Assistência à Saúde NOAS-SUS 01/2001. Diário Oficial da União, Poder Executivo, Brasília, DF, fev. 2001.

2. Algranti E, De Capitani EM, Carneiro APS, et al. Patologia respiratória relacionada com o trabalho. Patologia do trabalho 2003;2:1329-97.

3.Capitani EMD, Algranti E. Other pneumoconioses. Jornal Brasileiro de Pneumologia 2006; 32(2):54-59.

4. Cho $\mathrm{CH}$, Chiu NC, Ho CS, et al. Carbon monoxide poisoning in children. Pediatria Neonatal 2008; 49(4):121-5.

5. Hampson SE, Goldberg LR, Vogt TM, et al. Mechanisms by which childhood personality traits influence adult health status: educational attainment and healthy behaviors. Health psychology 2007; 26(1):121-125.

6. Khadem-Rezaiyan M, Afshari R. Carbon monoxide poisoning in Northeast of Iran. Journalofforensic and legal medicine 2016; 41:1-4.

7. Sarmento GJV. O ABC da fisioterapia respiratória. 1 ed. Manole: São Paulo, 2009.

8. Instituto Nacional do Câncer - INCA. Tabagismo: dados e números, 2007. Disponível em: <http://www.inca.gov.br>. Acessado em: 20 de junho, 2016.

9. Chen GD. Effect of hipoxia on noise-induced auditory impairment. Hearing Research 2002;172(1-2):186-95. 
10. Jarvis MJ, Belcher M, Vesey C, Hutchinson DCS.Monitoramento de monóxido de carbono de baixo custo em avaliação de tabagismo. Thorax 1986; 41(11):886-887.

11. Stewart RD, Stewart RS, Stamm W, et al.Estimativa rápida de níveis de carboxihemoglobina e combatentes contra-incêndio. Jama1976; 235(4):390-392.

12. American Thoracic Society - ATS. ATS Statement: guidelines for the six-minute walk test. AmericanJournal of Respiratory andCritical Care Medicine 2002; 166(1):11117.

13. Neder JA, Andreoni S, Lerario MC, et al. Reference values for lung function tests.II. Maximal respiratory and voluntary ventilation.Brazilian Journal of Medical Biological Research 1999; 32(6):719-27.

14. Ziegler B, Rovedder PME, Oliveira CL, et al. Predictors of oxygen desaturation during the six-minute walk test in patients with cystic fibrosis. Jornal Brasileiro de Pneumologia 2009; 35(10):957-965.

15. Enrigth PI, Sherril DI. Reference equations for the six minute walk in healthy adults. American Journal of Respiratory and Critical Care Medicine Home 1998; 158(5-1):13841387.

16. Moreira MAC, Moraes MR, Tannus R. Teste da Caminhada de Seis Minutos em Pacientes com DPOC durante Programa de Reabilitação. Jornal de Pneumologia2001; 27(6):295-300.

17. Javors MA, Hatch JP, Lamb RJ. Cut-off levels for breath carbon monoxide as a marker for cigarette smoking. Addiction Journal 2005; 100(2):159-67.

18. Santos UP, Gannam S, Abe JM, et al. Emprego da determinação de monóxido de carbono no ar exalado para a detecção do consumo de tabaco. Jornal de Pneumologia $2001 ; 27(5): 7-12$.

19. Peres FF. Meio Ambiente e Saúde: os efeitos fisiológicos da poluição do ar no desempenho físico - o caso do monóxido de carbono (CO). Arquivos em Movimento.2005;1(1):55-63.

20. Matielo Junior E, Gonçalves A. A corrida para a saúde: poluição ambiental no coração do problema. Revista Brasileira de Ciências do Esporte 1997; 18(2):111-118. 
21. Middleton ET, Morice AH. Breath carbon monoxide as an indication of smokinghabit. CHEST 2000; 117(3):758-763.

22. Rebagliato M. Validation of self reported smoking: editorial. Journal of Epidemiology \&Community Health2002; 56(3):164-165.

23. Fiore MC, Jáen CR, Baker TB, et al. Treating tobacco use and dependence: 2008 Update. Clinical Practice Guideline. Rockville, MD: Public Health Service, 2008.

24. Reichert J,Araújo AJ,Gonçalves CM, et al. Brazilian smoking cessation guidelines 2008.Jornal Brasileiro de Pneumologia 2008; 34(10):845-80.

25. Raw M, Anderson P, Batra A, et al. WHO Europe evidence based recommendations on the treatment of tobacco dependence.TobaccoControl 2002; 11(1):44-6.

26. Oliveira MV, Oliveira TR, Pereira CA, et al. Smoking among hospitalized patients in a general hospital. Jornal Brasileiro de Pneumologia 2008; 34(11):936-41. 